\title{
ASSOCIATION BETWEEN COGNITIVE FUNCTION AND CLUSTERED CARDIOVASCULAR RISK OF METABOLIC SYNDROME IN OLDER ADULTS AT RISK OF COGNITIVE DECLINE
}

\author{
M.M.Y. LAI ${ }^{1,2,3}$, D.J. AMES ${ }^{1}$, K.L. COX ${ }^{4}$, K.A. ELLIS ${ }^{1}$, M.J. SHARMAN ${ }^{5}$, G. HEPWORTH ${ }^{8}$, P. \\ DESMOND $^{7}$, E.V.CYARTO ${ }^{1,8}$, C. SZOEKE $^{9}$, R. MARTINS $^{10,11}$, \\ C.L. MASTERS ${ }^{12}$, N.T. LAUTENSCHLAGER ${ }^{1,13,14}$
}

\begin{abstract}
1. Academic Unit for Psychiatry of Old Age, Department of Psychiatry, The University of Melbourne, Melbourne, Australia; 2. South Metropolitan Health Service, Perth, Australia; 3. Curtin Medical School, Curtin University, Perth, Australia; 4. Medical School, The University of Western Australia, Perth, Australia; 5. School of Health Sciences, University of Tasmania, Launceston, Tasmania, Australia; 6. Statistical Consulting Centre, The University of Melbourne, Melbourne, Australia; 7. Department of Radiology, Royal Melbourne Hospital, The University of Melbourne, Australia; 8. Bolton Clarke Research Institute, Melbourne, Australia; 9. Department of Medicine, Royal Melbourne Hospital, The University of Melbourne, Melbourne, Australia; 10. School of Medical Sciences, Edith Cowan University, Perth, Australia; 11. Macquarie University, NSW, Australia; 12. The Florey Institute, The University of Melbourne, Australia; 13. WA Centre for Health \& Ageing, Medical School, University of Western Australia, Perth, Australia; 14. NorthWestern Mental Health, Melbourne Health, Melbourne, Australia. Corresponding author: Michelle M.Y. Lai, South Metropolitan Health Service, Locked Bag 100 Palmyra DC, WA 6961, Australia, Tel: +61 (08) 8152 8205, Fax: +616316 3318, Email: michelle.m.lai@curtin.edu.au
\end{abstract}

\begin{abstract}
Objectives: Metabolic syndrome (MetS) represents a cluster of obesity and insulin resistance-related comorbidities. Abdominal obesity, hypertension, elevated triglyceride and glucose levels are components of MetS and may have a negative effect on cognitive function, but few cognitive studies have examined the combined risk severity. We sought to determine which specific cognitive abilities were associated with MetS in older adults at risk of cognitive decline. Design: Cross-sectional study. Participants: 108 AIBL Active participants with memory complaints and at least one cardiovascular risk factor. Measurements: Cardiovascular parameters and blood tests were obtained to assess metabolic syndrome criteria. The factors of MetS were standardized to obtain continuous z-scores. A battery of neuropsychological tests was used to evaluate cognitive function. Results: Higher MetS z-scores were associated with poorer global cognition using ADAS-cog (adjusted standardized beta $=0.26$, SE 0.11, p $<0.05$ ) and higher Trail Making B scores (adjusted beta=0.23, SE 0.11, $\mathrm{p}<0.05)$. Higher MetS risk was related to lower cognitive performance. Conclusion: Combined risk due to multiple risk factors in MetS was related to lower global cognitive performance and executive function. A higher MetS risk burden may point to opportunities for cognitive testing in older adults as individuals may experience cognitive changes.
\end{abstract}

Key words: Metabolic syndrome, cognitive function, cardiovascular risk, obesity.

\begin{abstract}
Abbreviations: ADAS-cog: Alzheimer's disease Assessment Scale-Cognitive Section; AIBL: Australian Imaging Biomarkers \& Lifestyle; BP: blood pressure; CERAD: Consortium to Establish a Registry for Alzheimer's Disease; HDL-C: high-density lipoprotein-cholesterol; LR: likelihood ratio; MetS: metabolic syndrome; MMSE: mini-mental state examination; NCEP-ATP: National Cholesterol Education Program Adult's Treatment Panel; TAG: triglyceride; TMT: trail making test.
\end{abstract}

\section{Introduction}

Obesity-related comorbidities such as hypertension, type II diabetes and hypertriglyceridemia are linked to increased risk of cognitive decline and dementia (1), but an understanding of the combined risk due to multiple risk factors on cognitive health is limited. Metabolic syndrome (MetS) is a clinical entity associated with underlying insulin resistance with a cluster of at least three of five cardiovascular risk factors (central obesity, hypertension, hypertriglyceridemia, HDL-cholesterol (HDLC) and impaired glucose metabolism) (2). Early identification and treatment of individuals with MetS at risk of cognitive impairment may be of significant value in reducing the risk of metabolic and cognitive decline (1). However, the nature of the association between MetS and cognition is unclear (3), whether the combined risk of concurrent metabolic disorders in MetS has a negative impact on specific cognitive function in older persons (4). A French study found that hypertriglyceridemia, low HDL-C, and diabetes, but not elevated fasting glucose, were associated with a steeper cognitive decline (5), suggestive that the clustered cardiovascular risk might have an important association with cognitive performance.

Current evidence in studying the relationship between MetS and cognition is inconclusive in part due to the use of clinical measures for MetS in research that provides no information on risk severity (6). The dichotomous classification of the presence of the clinical syndrome uses arbitrary cut-offs for the components (i.e., the presence or absence of central abdominal obesity, hypertriglyceridemia, reduced high-density lipoprotein, hypertension and impaired fasting glucose) (2). While the dichotomous clinical definition of the presence or absence of MetS has limitations, small changes in the component values could result in a change in the clinical status of having or not having MetS, particularly in individuals having borderline 
values and multiple components of the syndrome. One approach to addressing the clustering effect of cardiovascular risk factors in epidemiological studies is statistical z-score standardization to construct a continuous risk estimate (6).

Because of subclinical manifestation of dementia before the diagnosis, estimating the risk of MetS in older people at risk of cognitive deterioration might provide an opportunity for early detection and insight into new avenues for diseasecourse modification (1). This study hypothesized that MetS risk severity is related to cognitive performance in adults with memory complaints. The objective was to identify the cognitive patterns in relation to MetS using the clinical criteria, its associated risk severity using $\mathrm{z}$-scores and increasing numbers of components.

\section{Materials and Methods}

This study analysed the baseline data of the Australian Imaging Biomarkers \& Lifestyle (AIBL) Active randomized controlled trial study participants, who were communitydwelling older adults aged over 60 years with mild cognitive impairment (MCI) or subjective memory complaints and at least one cardiovascular risk factor including physical inactivity, obesity, hypertension, heart disease, diabetes, smoking and dyslipidemia (7). The AIBL Active trial investigated whether a 24-month physical activity program can delay the progression of white matter changes on neuroimaging (8) and the trial participants were recruited from the AIBL flagship study, a multidisciplinary prospective study of aging previously described (9). Those with a diagnosis of dementia based on Mini-Mental State Examination (MMSE) (10), depression at baseline and severe visual or hearing impairment were not included (7). All participants provided written consent. Ethics approval for AIBL Active study analyses was obtained from the Melbourne Health Human Research Ethics Committee.

Trained research assistants overseen by neuropsychologists administered a battery of neurocognitive tests at baseline to assess global cognition, verbal memory and executive functioning as per the protocol paper (7). An MMSE (0-30) score of less than 24 excluded participants from the study (10). Lower MMSE and higher Alzheimer's disease Assessment Scale-Cognitive Section (ADAS-cog, 0-70) (11) scores indicate increased severity of global cognitive impairment. The Consortium to Establish a Registry for Alzheimer's Disease (CERAD) tool (12) was used for classification of MCI. The individual tests of CERAD in verbal fluency and delayed recall were used to assess executive function and verbal memory respectively. Trail making tests (TMT) A \& B are two parts of an executive function test that provides information about visual scanning, processing speed and mental flexibility.

Waist circumference was measured three times, according to the standardized protocol. Resting seated blood pressure (BP) was assessed as the mean of five measurements at two-minute intervals using a digital monitor. For cardiovascular health and medications, a physician-researcher adjudicated the medical records obtained from each participant's doctor. Blood samples for analysis of vascular risk factors were collected at baseline and analyzed at a certified laboratory (Melbourne Pathology, VIC). The National Cholesterol Education Program Adult's Treatment Panel (NCEP-ATP) III clinical definition of MetS was used (2).

All analyses were conducted using Stata (v23.1 Stata Corps). Standardized z-scores were constructed with lower scores indicating a favorable MetS risk profile. The construct is consistent with the research practice according to the American Diabetes Association and the European Association for the Study of Diabetes (6). The z-score was defined as the sum of z-scores of the waist, BP, fasting HDL-C, triglycerides and glucose. The HDL-C z-scores were multiplied by -1 for the inverse protective relationship with cardiovascular risk (6). The resulting z-score combines individual components for a person and represents the combined risk burden.

Table 1

Characteristics of participants with and without metabolic syndrome

\begin{tabular}{|c|c|c|c|c|}
\hline \multirow[t]{3}{*}{ Variables $($ mean \pm SD) } & \multicolumn{4}{|c|}{ Metabolic syndrome } \\
\hline & \multicolumn{2}{|c|}{ Yes $(n=31)$} & \multicolumn{2}{|c|}{ No $(n=77)$} \\
\hline & mean & SD & mean & SD \\
\hline Age (years) & 73.4 & 5.0 & 73.1 & 6.1 \\
\hline Female, n (\%) & 13 & 41.9 & 44 & 57.1 \\
\hline Smoking history,n (\%) & 12 & 40 & 28 & 36.4 \\
\hline Education (years) & 13.6 & 3.2 & 14.3 & 3.8 \\
\hline Waist (cm) & 100.1 & 14.1 & 86.8 & 11.0 \\
\hline Systolic blood pressure (mmHg) & 126.0 & 15.5 & 126.7 & 15.7 \\
\hline Diastolic blood pressure $(\mathrm{mmHg})$ & 71.5 & 7.7 & 72.6 & 8.9 \\
\hline Triglyceride (mg/dL) & 1.7 & 0.8 & 1.1 & 0.4 \\
\hline $\mathrm{HDL}-\mathrm{C}(\mathrm{mg} / \mathrm{dL}) \dagger$ & 1.3 & 0.4 & 1.6 & 0.4 \\
\hline LDL-C $(\mathrm{mg} / \mathrm{dL}) \ddagger$ & 2.8 & 1.3 & 3.2 & 0.8 \\
\hline Cholesterol (mg/dL) & 4.9 & 1.4 & 5.3 & 0.9 \\
\hline Glucose (mg/dL) & 5.8 & 1.2 & 5.2 & 1.1 \\
\hline \multicolumn{5}{|l|}{ Treatment for } \\
\hline Hypertension, n (\%) & 22 & 71.0 & 25 & 32.5 \\
\hline Diabetes Mellitus, n (\%) & 6 & 19.4 & 3 & 3.9 \\
\hline Dyslipidemia, n (\%) & 21 & 67.7 & 27 & 35.1 \\
\hline Metabolic syndrome (z) & 2.2 & 2.6 & -1.0 & 2.4 \\
\hline
\end{tabular}

Cognitive test scores were compared between the two groups (with and without MetS) using independent t-tests. The associations of each cognitive test and MetS were assessed in separate univariate regression models. The analyses were repeated using multivariate regression to adjust for age, gender 
THE JOURNAL OF NUTRITION, HEALTH \& AGING@

Table 2

Correlation coefficients between global cognition (ADAS-cog) according to metabolic syndrome (MetS) and its component factors (z-scores) $(\mathrm{n}=108)$

\begin{tabular}{lcccccc}
\hline \multicolumn{1}{c}{ Risk factors } & \multicolumn{5}{c}{ Metabolic Syndrome Factors } \\
Variables & MetS (z) & Waist (z) & BP (z) & TAG (z) & HDL-C (z) & Glucose (z) \\
\hline Age (years) & 0.13 & 0.05 & $0.19^{*}$ & 0.07 & -0.04 & 0.04 \\
Education (year) & -0.05 & -0.06 & 0.11 & -0.03 & 0.05 & -0.14 \\
Global cogntion (ADAS-cog) & $0.26^{*}$ & $0.20^{*}$ & 0.07 & -0.03 & -0.17 & $0.28^{*}$ \\
\hline
\end{tabular}

MetS: metabolic syndrome, TAG: triglycerides, HDL-C: high-density lipoprotein-cholesterol. ADAS-cog: Alzheimer's Disease Assessment Scale-Cognitive Subscale

Table 3

Standardized estimates of separate multivariate linear regression models showing the relationship between cognitive tests (dependent variable) and metabolic syndrome (MetS) (independent variable), adjusted for age, gender and education in years

\begin{tabular}{llccccccc}
\hline Cognitive tests $\$$ & & MMSE & ADAS-cog & CDR & TMT A & TMT B & Verbal Fluency & Delayed recall \\
\hline MetS $\dagger$ Model & $\mathrm{n}$ & 106 & 106 & 106 & 103 & 103 & 106 & 106 \\
1. Clinical criteria $¥(\mathrm{y} / \mathrm{n})$ & $\beta$ & 0.09 & 0.06 & -0.09 & -0.11 & 0.16 & 0.02 & -0.03 \\
& $\mathrm{SE}$ & 0.10 & 0.10 & 0.09 & 0.10 & 0.10 & 0.10 & 0.10 \\
2. Risk burden $(\mathrm{z}-\mathrm{score})$ & $\beta$ & -0.07 & $0.22^{*}$ & 0.06 & 0.11 & $0.25^{*}$ & 0.08 & -0.20 \\
& $\mathrm{SE}$ & 0.11 & 0.10 & 0.10 & 0.11 & 0.11 & 0.11 & 0.11 \\
3. Number of MetS $\dagger$ factors & $\beta$ & 0.13 & 0.08 & -0.08 & -0.01 & $0.21^{*}$ & 0.02 & -0.12 \\
& $\mathrm{SE}$ & 0.10 & 0.10 & 0.09 & 0.10 & 0.10 & 0.10 & 0.10 \\
\hline
\end{tabular}

$* \mathrm{P}<0.05 . \dagger$ MetS: MetS; $\$$ MetS clinical status according to the National Cholesterol Education Program Adult Treatment Panel (NCEP-ATP) III criteria coded as $0 / 1$ for yes/no; $\$$ MMSE: Mini-Mental Status Examination, ADAS-cog: Alzheimer's Disease Assessment Scale-Cognitive Subscale, CDR: Clinical Dementia Rating Scale; TMT: Trail Making Test.

and education. Likelihood ratio (LR) tests compared the models of fit to assess the quality of the models using MetS measures as continuous (z-score) or discrete (number of components). MetS entered the multivariate regression models according to the presence or absence of MetS clinical status (model 1), continuous MetS z-scores (model 2) and the total number of MetS components (model 3). Each cognitive test was fitted separately to the models. It was determined that a sample size of 108 would provide approximately $80 \%$ power to detect a two-point difference between groups with a significance level 0.05 based on SD 3.5 in ADAS-cog and the national estimates of cardiovascular factor clustering $(>3)$ of prevalence about $30 \%(13)$.

\section{Results}

MetS was observed in 31 participants $(28.7 \%)$. The characteristics of the participants are shown in Table 1. There were no inter-group differences in cognitive performance $(\mathrm{p}$ $>0.05$ ) (not shown). ADAS-cog had a weak positive correlation with age $(\mathrm{r}=0.19, \mathrm{p}<0.05)$, a negative correlation with education in years $(\mathrm{r}=-0.26, \mathrm{p}<0.01)$. It also had a moderate positive correlation with MetS $(\mathrm{r}=0.26, \mathrm{p}<0.05))$ as well as its components of waist circumference $(r=0.20, p<0.05)$ and fasting glucose $(\mathrm{r}=0.28, \mathrm{p}<0.05)$ but had no significant correlations with BP, triglycerides and HDL-C (table 2). MetS $\mathrm{Z}$-scores were significantly associated with gender (M:1.39 v $\mathrm{F}: 1.32, \mathrm{p}<0.001)$.

In the univariate analyses (not shown), MetS z-scores had significant associations with poorer global cognition, with higher ADAS-cog (beta $=0.35, \mathrm{p}<0.01)$ and lower CERAD delayed recall (beta $=-0.19, \mathrm{p}<0.01$ ). In the multivariate analyses (table 3), MetS clinical status was not significantly associated with performance on cognitive tests. A higher z-score was associated with poorer global cognition, with higher ADAS-cog (adjusted beta $=0.26, \mathrm{p}<0.05$ ) and TMT B (adjusted beta $=0.23$, $\mathrm{p}<0.05)$ after adjustment for covariates. An increasing number of MetS components was significantly associated with higher TMT B (adjusted beta $=0.20, \mathrm{p}<0.05$ ). There was no evidence that the association between cognitive measures and MetS continuous model (z-scores) was a better fit than the discrete model (number of components) (LR tests $\mathrm{p}<0.05$ ). Omitting one outlier in TMT B did not change the significance of the associations. Residual analyses for the normality assumptions of the regression models were deemed acceptable. 


\section{Discussion}

This research highlighted the relevance of the risk burden in MetS and its association with cognitive performance in the study participants who were older adults with memory complaints or mild cognitive impairment. Abdominal obesity and fasting glucose levels were components of MetS and had positive correlations with reduced global cognition. The relationship between the global cognition and z-scores in our participants is consistent with the longitudinal Three-City Study (5), in which MetS was associated with a decline in global cognition, although this French study has a lower MetS prevalence of $15.8 \%$ using NCEP-ATP III definition. The relationship between TMT B and MetS (z-scores and number of components) in our findings is consistent with a Korean study, in which significantly lower TMT B scores were observed in older participants with MetS (14). TMT is sensitive in detecting inefficiencies in psychomotor speed and executive function, relating to frontal lobe functions that may reflect the initial cognitive changes in the prodromal stage of vascular-related pre-dementia.

Our study sample is representative of the general Australian population concerning cardiovascular risk, with a similar prevalence of MetS affecting one-third of the general population (13). The standardized continuous variable of MetS was more sensitive to cognitive associations than the presence or absence of MetS variable. A continuous z-score or ordered categorical measure would more closely approximate individuals' actual continuum of risk when compared with the dichotomous clinical status of the syndrome (6). The adjusted unstandardized coefficient for ADAS-cog of 0.35 indicates that improving cognitive function and decreasing the ADAS-cog by 3.5 points would arise from a lower vascular burden of MetS z-score by 0.1 units.

Mechanisms underlying cognitive deficits in MetS may be beyond atherosclerosis, contributing to microvascular and macrovascular disease. Current views suggest that MetS is a multiplex risk factor arising from insulin resistance and abnormal adipose deposition and is associated with vascular endothelial damage and reduces cerebrovascular blood flow that may increase the risk of cognitive impairment (15). Insulin resistance and hyperinsulinemia decrease glucose use and energy metabolism in the cerebral cortex and may contribute to amyloid deposits and phosphorylation of tau protein (16). Cholesterol is a significant component of cell membranes and myelin. Modification of cholesterol distribution in the membrane appears to decrease this membrane fluidity in the hippocampus, the memory centre affected early in Alzheimer's disease (17).

This study provides comprehensive, objective measures, as opposed to a single screening test to indicate cognitive function. The result may be applied to community-dwelling older adults at high risk of cognitive decline with one or more cardiovascular risk factors, but not to people with dementia and healthy adults without cognitive complaints, patients with depression, severe hearing loss or visual impairment, because they were excluded from the study. The cross-sectional study design and the possibility of residual confounding limit the inference of causality between cognition, protective factors and cardio-metabolic risk factors.

\section{Conclusions}

MetS risk was associated with poorer global cognitive performance and executive function. A higher MetS risk burden in patients might prompt clinicians to consider cognitive testing as individuals might experience cognitive changes. Further prospective studies should explore its predictive value in vascular cognitive impairment and determine the mechanisms linking them.

Acknowledgment: We thank the Australian Imaging Biomarker and Lifestyle Study of Ageing participants for their participation and the completion of the study, the participating research staff at the National Ageing Research Institute (NARI) and the Mental Health Research Institute (MHRI) for the collection of physical assessments and the administration of the study. This project is supported by a project grant from Australia's National Health and Medical Research Council Centre of Research Excellence in Cognitive Health (APP1100579).

Statement of Ethics: Subjects have given their written informed consent. Ethics approval for the AIBL Active study protocol and analyses was obtained from the Melbourne Health Human Research Ethics Committee.

Disclosure Statement: The authors have no conflict of interest to declare.

Funding Sources: This project is supported by a project grant from Australia's National Health and Medical Research Council Centre of Research Excellence in Cognitive Health (APP1100579). Trial Registration: Australia New Zealand Clinical Trials Registry ACTRN12611000612910

\section{References}

1. Panza F, Frisardi V, Capurso C, Imbimbo BP, Vendemiale G, Santamato A, et al Metabolic syndrome and cognitive impairment: current epidemiology and possible underlying mechanisms. J Alzheimers Dis. 2010;21(3):691-724.

2. NCEP-ATPIII. Third report of the National Cholesterol Education Program (NCEP) expert panel on detection, evaluation, and treatment of high blood cholesterol in adults (Adult Treatment Panel III) final report. Circulation. 2002;106(25):3143-421.

3. Assuncao N, Sudo FK, Drummond C, de Felice FG, Mattos P. Metabolic Syndrome and cognitive decline in the elderly: A systematic review. PLoS One. 2018;13(3):e0194990.

4. Vieira JR, Elkind MS, Moon YP, Rundek T, Boden-Albala B, Paik MC, et al. The metabolic syndrome and cognitive performance: the Northern Manhattan Study. Neuroepidemiology. 2011;37(3-4):153-9.

5. Raffaitin C, Feart C, Le Goff M, Amieva H, Helmer C, Akbaraly TN, et al. Metabolic syndrome and cognitive decline in French elders: the Three-City Study. Neurology. 2011;76(6):518-25.

6. Carroll SJ, Paquet C, Howard NJ, Adams RJ, Taylor AW, Daniel M. Validation of continuous clinical indices of cardiometabolic risk in a cohort of Australian adults. BMC cardiovascular disorders. 2014;14:27.

7. Cyarto EV, Lautenschlager NT, Desmond PM, Ames D, Szoeke C, Salvado O, et al. Protocol for a randomized controlled trial evaluating the effect of physical activity on delaying the progression of white matter changes on MRI in older adults with memory complaints and mild cognitive impairment: the AIBL Active trial. BMC Psychiatry. 2012;12:167.

8. Venkatraman VK, Sanderson A, Cox KL, Ellis KA, Steward C, Phal PM, et al. Effect of a 24-month physical activity program on brain changes in older adults at risk of Alzheimer's Disease: the AIBL Active trial. Neurobiology of Aging. 2019.

9. Ellis KA, Bush AI, Darby D, De Fazio D, Foster J, Hudson P, et al. The Australian Imaging, Biomarkers and Lifestyle (AIBL) study of aging: methodology and baseline characteristics of 1112 individuals recruited for a longitudinal study of Alzheimer's disease. Int Psychogeriatr. 2009;21(4):672-87. 


\section{THE JOURNAL OF NUTRITION, HEALTH \& AGINGC}

10. Folstein MF, Folstein SE, McHugh PR. "Mini-mental state". A practical method for grading the cognitive state of patients for the clinician. Journal of psychiatric research. 1975;12(3): 189-98.

11. Rosen WG, Mohs RC, Davis KL. A new rating scale for Alzheimer's disease. Am J Psychiatry. 1984;141(11):1356-64.

12. Welsh KA, Butters N, Mohs RC, Beekly D, Edland S, Fillenbaum G, et al. The Consortium to Establish a Registry for Alzheimer's Disease (CERAD). Part V. A normative study of the neuropsychological battery. Neurology. 1994;44(4):609-14.

13. AIHW. Heart, stroke and vascular diseases - Australian facts 2004. AIHW Cat. No. CVD 27. Canberra: Australian Institute of Health and Welfare (AIHW) and National Heart Foundation of Australia (Cardiovascular Disease Series No. 22); 2004.
14. Oh HM, Kim SH, Kang SG, Park SJ, Song SW. The relationship between metabolic syndrome and cognitive function. Korean journal of family medicine. 2011;32(6):35866.

15. Arshad N, Lin TS, Yahaya MF. Metabolic Syndrome and Its Effect on the Brain: Possible Mechanism. CNS Neurol Disord Drug Targets. 2018;17(8):595-603.

16. Hoyer S. The brain insulin signal transduction system and sporadic (type II) Alzheimer disease: an update. Journal of neural transmission. 2002;109(3):341-60.

17. Eckert GP, Cairns NJ, Maras A, Gattaz WF, Muller WE. Cholesterol modulates the membrane-disordering effects of beta-amyloid peptides in the hippocampus: specific changes in Alzheimer's disease. Dement Geriatr Cogn Disord. 2000;11(4):181-6. 